\title{
Castration affects reproductive but not aggressive behavior in a cichlid fish
}

\author{
Olinda Almeida ${ }^{a}$, Adelino V.M. Canário ${ }^{\mathrm{b}}$, Rui F. Oliveira ${ }^{\mathrm{a}, \mathrm{c}, *}$ \\ a Unidade de Investigação em Eco-Etologia, ISPA - Instituto Universitário, Rua Jardim do Tabaco, 34, 1149-041 Lisboa, Portugal \\ b CCMAR - Centro de Ciências do Mar, Universidade do Algarve, Gambelas, 8005-139 Faro, Portugal \\ ' Champalimaud Neuroscience Programme, Instituto Gulbenkian de Ciência, Rua da Quinta Grande 6, 2780-156 Oeiras, Portugal
}

\section{A R T I C L E I N F O}

Article history:

Available online $\mathrm{xxxx}$

\section{Keywords:}

Tilapia

Castration

Gonadal hormones

Androgens

Aggression

Courtship

\begin{abstract}
A B S T R A C T
Gonads are the main source of sex steroids, which have been implicated in the regulation of sexually differentiated behavior, such as reproductive and aggressive displays. In the Mozambique tilapia (Oreochromis mossambicus) territorial males have higher androgen levels than non-territorials, express reproductive behavior and use a urine-borne pheromone to signal their social status towards conspecifics. Here we investigated the effects of gonadectomy on the circulating levels of androgens and cortisol, and on the expression of aggressive and reproductive behavior (nest building, courtship behavior, and nuptial coloration). Males were either castrated, urine bladder damaged, or sham-operated and visually exposed to a group of females during 8 consecutive days and subsequently to a male on day 9 . The urine bladder damaged treatment was included in the experimental design because a full castration procedure in this species causes quite often damage to the urine bladder. Gonadectomy lowers dramatically the circulating levels of androgens measured at 4 and 8 days post-castration and abolishes the expression of nest building, courtship behavior and nuptial coloration, but has no effect on the expression of aggressive behavior. These results confirm the gonads as the main source of androgens in this species and show that androgens are necessary for the expression of reproductive behaviors. However, the expression of aggressive behavior seems to be decoupled from gonadal steroids, namely androgens, suggesting the action of independent central mechanisms.
\end{abstract}

(c) 2014 Published by Elsevier Inc.

\section{Introduction}

The gonads are the main source of sex hormones, which have a pivotal role in sex differentiation, the development of secondary sex characters and the expression of sex specific behaviors (Nelson, 2005). Therefore, gonadal hormones have been viewed as playing an integrative role that assures the co-expression of sets of functional traits in the same phenotype, such that differentiation of the gonads into a given sex express implies the expression of the corresponding secondary sexual characters and behavior. In males, androgens play such a role, either directly or through aromatization into estrogens (Balthazart and Ball, 1998). Androgens have been implicated in the expression of multiple aspects of reproductive behavior including courtship displays and breeding-related aggression (e.g. defence of breeding territories or mates, Pfaff et al., 2008). However, the fact that androgens modulate the

\footnotetext{
* Corresponding author at: Unidade de Investigação em Eco-Etologia, ISPA Instituto Universitário, Rua Jardim do Tabaco, 34, 1149-041 Lisboa, Portugal.

E-mail address: ruiol@ispa.pt (R.F. Oliveira).
}

expression of sex-specific behavior does not demonstrate per se that they are necessary for its expression. In fact, a compilation of the effects of castration on reproductive and aggressive behavior across different fish species shows divergent results (see Oliveira and Gonçalves (2008), and Gonçalves and Oliveira (2010) for recent reviews). Castration reduced or abolished the expression of reproductive behaviors, such as nest building, nuptial coloration or courtship displays, in some species, whereas it had no effect in others (Oliveira and Gonçalves, 2008; Gonçalves and Oliveira, 2010). Similarly, the effects of castration on aggressive behavior ranged from negative, to neutral, and even to positive (Oliveira and Gonçalves, 2008; Gonçalves and Oliveira, 2010), and divergent results can be found within the same species (e.g. Gasterosteus aculeatus; Hoar, 1962; Baggerman, 1966; Wootton, 1970).

Cichlid fishes have been emerging as model organisms in behavioral neuroendocrinology, given the complexity of their social and breeding behavior (e.g. cooperative breeding in Neolamprologus pulcher, Desjardins et al., 2007; Fitzpatrick, 2005; Taves et al., 2009; Wong and Balshine, 2011) and the diversity of mating systems and parental care types present in closely related species 
that allows for comparative studies (e.g. phylogenetic test of the challenge hypothesis in African cichlids with divergent mating systems, Hirschenhauser et al., 2004). In our lab we have been using the Mozambique tilapia (Oreochromis mossambicus) as a model to study the neuroendocrinology of social behavior. In this species androgens respond to social interactions (Oliveira et al., 1996) and socially driven changes in androgens moderate the expression of both secondary sex characters (Oliveira and Almada, 1998) and male-male competitive behavior (i.e. winner effect, Oliveira et al., 2009). Therefore it became important to assess if androgens are necessary for the expression of reproductive and aggressive behaviors, which would suggest an activational role of androgens on the neural circuits underlying behavior, or if they act as moderators, which would be compatible with a neuromodulator role (e.g. facilitator) on a neural circuit that would be functional even in the absence of androgens.

Here the effects of castration on reproductive behavior (nuptial coloration, nest building and courtship displays) expressed in the presence of females, and on aggressive behavior displayed towards a neighboring male were tested in male 0 . mossambicus. Given that the complete excision of the gonads in this species involves damage to the urinary bladder (see details in the Methods Section 2.3), and the fact that male 0 . mossambicus use urine-born compounds to communicate social status (Almeida et al., 2005; Barata et al., 2007), the effects of urinary bladder damage were also investigated. In summary, the specific aims of this study were: (1) to characterize the effects of castration in circulating levels of androgens (testosterone, T; and 11-keto-testosterone, KT); (2) to investigate the effects of castration on reproductive and aggressive behaviors; and (3) to verify the effects of urinary bladder damage on circulating levels of androgens, and on aggressive and courtship behavior.

\section{Materials and methods}

\subsection{Animals and housing}

The tilapia males used in this study were part of a stock held at ISPA - Instituto Universitário (Lisboa, Portugal) that is maintained in glass tanks $(120 \times 40 \times 50 \mathrm{~cm}, 240 \mathrm{~L})$ with a fine gravel substrate, in stable social groups of 4 males and 5 females per tank. Tanks were supplied with a double filtering system (sand and external biofilter, Eheim) and constant aeration. Water quality was monitored on a weekly basis for nitrite $(0.2-0.5 \mathrm{ppm})$, ammonia $(<0.5 \mathrm{ppm})$ (Pallintest $\left.\mathrm{kit}^{\circledR}\right)$ and $\mathrm{pH}(6.0-6.2)$. Fish were kept at a temperature of $26 \pm 2{ }^{\circ} \mathrm{C}$ and a $12 \mathrm{~L}: 12 \mathrm{D}$ photoperiod, and fed with commercial cichlid floating and sinking sticks. The social status of the males in each tank, based on the body coloration and on the possession of a spawning pit on the substrate, was monitored on a daily basis.

\subsection{Experimental protocol}

Twenty-eight territorial males [territorial status was assessed from nuptial coloration and nest defence behavior; body mass ( mean $\pm \mathrm{SD}$ ): castrated $=41.4 \pm 7.0 \mathrm{~g}$; sham $=41.7 \pm 8.4 \mathrm{~g}$; urinary bladder damaged $=33.3 \pm 1.7 \mathrm{~g}$ ] were removed from the stock tanks and introduced in experimental aquaria $(47 \mathrm{~cm} \times 24 \mathrm{~cm} \times$ $30 \mathrm{~cm}$, following stock conditions) where they were left for 2 days in order to familiarize with the new aquarium (days -2 and -1 , see Fig. 1 for the time line of the general protocol). One to $3 \mathrm{~h}$ before males introduction in the experimental aquaria, 4 females [body mass (mean $\pm \mathrm{SD}$ ): $23.2 \pm 5.2 \mathrm{~g}, N=30$ )] were collected from stock tanks and introduced in an adjacent aquarium $(70 \mathrm{~cm} \times$ $37 \mathrm{~cm} \times 30 \mathrm{~cm}$ ) and acted as the visual stimulus to focal males. On day 0 males underwent one of three types of surgery: (1) sham operation; (2) castration; and (3) urinary bladder damage. The latter treatment was used as a complementary control treatment because the total removal of the testis implied damaging to the urinary bladder (Fig. 2). In order to prevent exposure of the experimental males to their own hormones, that might have been released into the water, the water of the experimental aquaria was renewed immediately after surgery and any nests built by the males on days $-2,-1$ and 0 (i.e. before surgery) were destroyed. Males were then returned to their experimental aquaria and kept in visual contact with females.

Blood was collected from the caudal vein (using $1 \mathrm{ml}$ syringes with 25G/16 mm needles) under anaesthesia (MS-222, Pharmaq; 300-400 ppm) at days 0 (before surgery), 4 and 8. Blood sampling always took less than 4 min since the induction of anaesthesia, which prevents any possible effects of handling stress on cortisol levels (Foo and Lam, 1993). Blood was centrifuged and the plasma was stored at $-20^{\circ} \mathrm{C}$ until further processing.

At day 8 an unfamiliar male to the focal male was placed isolated in an adjacent aquarium $(18 \mathrm{~cm} \times 30 \mathrm{~cm} \times 15 \mathrm{~cm})$ at the opposite side of the females' aquarium (the side of the male and female aquaria was randomized across replicates). During day 8 visual access between the two males was prevented by placing a white opaque partition between the aquaria. During day 9 visual access to females was blocked by insertion of a white opaque partition and visual contact to the male in the adjacent aquarium was allowed for $15 \mathrm{~min}$. The focal male behavior (bite, displays and courtship) towards the adjacent (putative intruder) male was

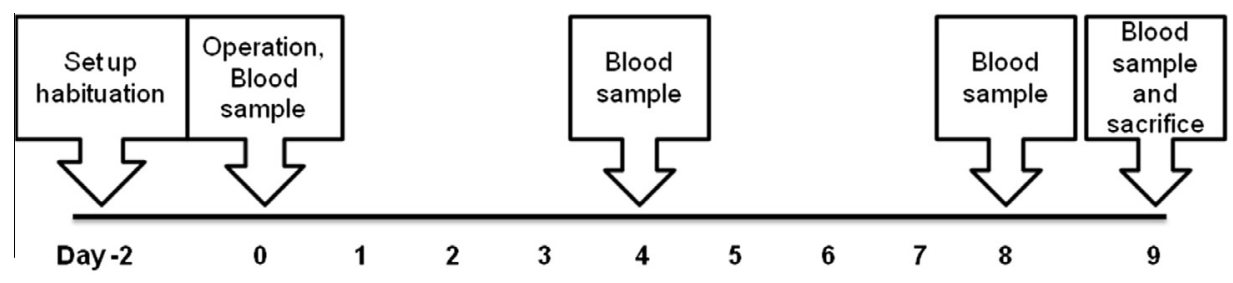

Behavioural observation (scan sampling): male inteacting with neighbour females (bite and courtship were recorded daily). Body coloration and nest existence registration in a daily basis.

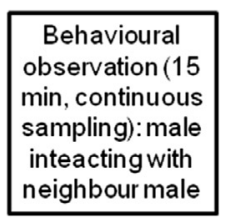

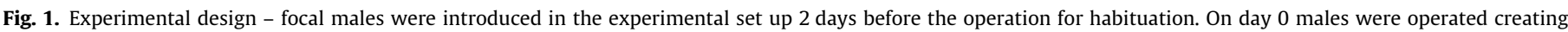

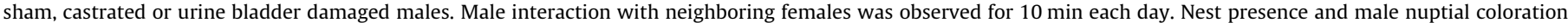

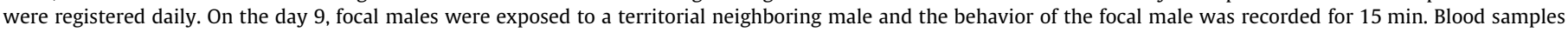
were collected on days 0 (before operation), 4 and day 8 - (after the interaction with the neighbor male). 

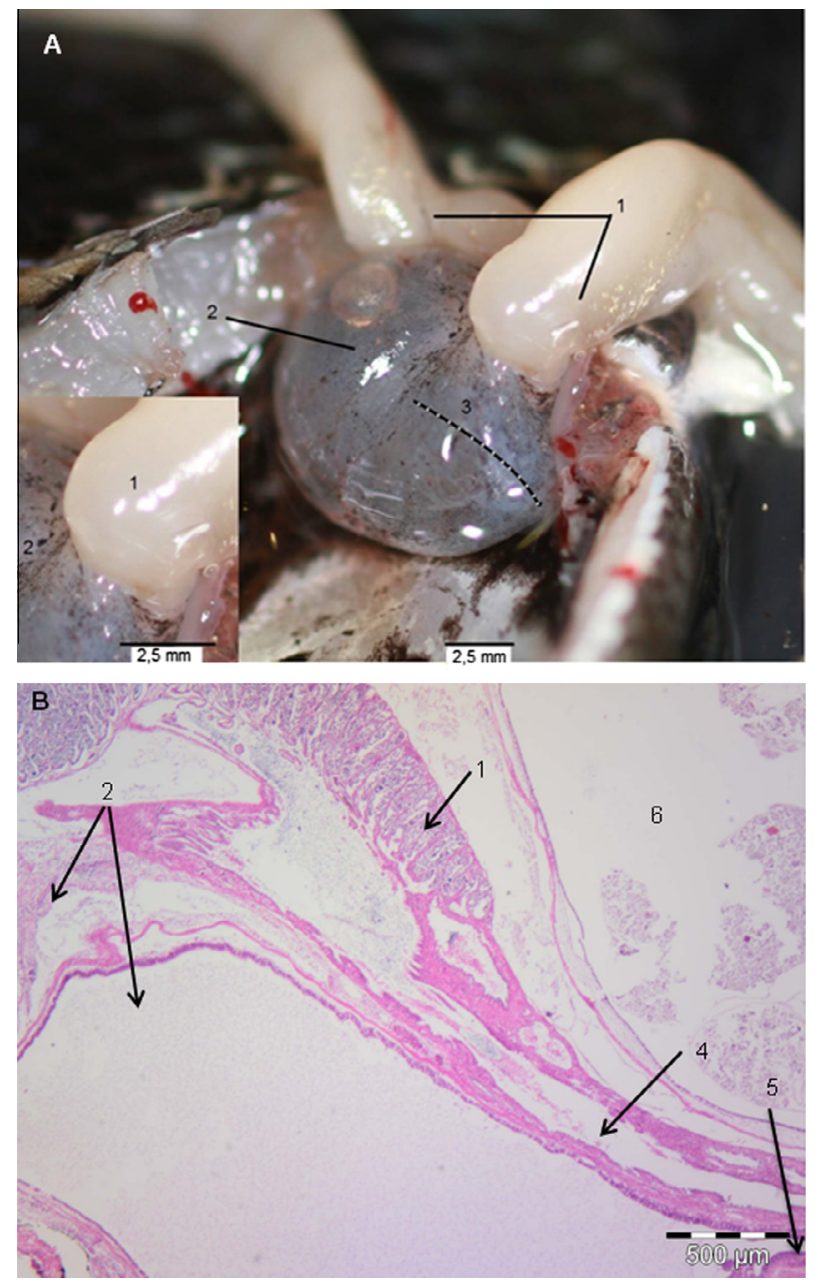

Fig. 2. Detail of the area of the junction between the gonads and the urinary bladder. The testis and the urinary bladder are juxtaposed in the proximal region of testis. To avoid leaving any vestigial gonadal tissue that could be a source of androgens, the urinary bladder is inevitably damaged during castration. (A) In the urinary bladder damaged treatment one incision on each side of the bladder was made (3, dashed line represents the local and the extension of the incision on the left side). (B) Histological section (magnification $40 \times$ ) to illustrate the proximity of the gonads and bladder that prevents a full castration to be performed without damage to the bladder: 1 - gonads; 2 - urinary bladder; 3 - local of incision on the left side of the urinary bladder; 4 - sperm duct; 5 - urethra and 6 - intestine.

recorded using a multievent recorder (The Observer v. 5, Noldus Technology). On day 9 , fish were sacrificed by decapitation under deep anaesthesia.

\subsection{Surgical procedures}

Fish were anaesthetized and placed belly-up in a "V" shaped bed with an oral supply of dechlorinated and aerated water containing anaesthetic (150-200 ppm). A ventral mid-sagittal incision was made from the pelvic fin region towards the anal region. Circa 3-4 $\mathrm{mm}$ from reaching the anus opening the direction of the incision was moved $120^{\circ}$ to the right and proceeded for ca. $0.5 \mathrm{~cm}$, in order to expose the insertion of the gonads. In the castrated treatment both testis were totally removed by a proximal incision. Given the tight insertion of the testicular ducts with the urinary bladder (Fig. 2), and to make sure that no vestigial testicular tissue remained, the urinary bladder was always partially severed as a result of the complete castration procedure. To control for this injury during castration a control treatment was included consisting of a partially sectioned urinary bladder only, in which 2 side cuts
Table 1

Repeated measures ANOVA analyses for androgen levels (testosterone. 11-ketotestosterone) using period of observation (day 0 vs. day 4 vs. day 8) as a repeated factor and treatment (sham operated vs. urinary bladder damaged vs. castrated) as a categorical factor. Results from a priori planned comparisons are also presented.

\begin{tabular}{|c|c|c|c|c|}
\hline \multirow[t]{2}{*}{ ANOVA } & \multicolumn{2}{|c|}{ Testosterone } & \multicolumn{2}{|c|}{ 11-Keto-testosterone } \\
\hline & $F$ & $p$-Value & $F$ & $p$-Value \\
\hline Period & 24.75 & $<0.001$ & 21.30 & $<0.001$ \\
\hline Treatment & 44.17 & $<0.001$ & 19.99 & $<0.001$ \\
\hline Period * treatment & 22.08 & $<0.001$ & 19.17 & $<0.001$ \\
\hline \multicolumn{5}{|l|}{ Planned comparisons } \\
\hline D0:SH-UBD & 3.34 & 0.079 & 0.10 & 0.760 \\
\hline D0:SH-CA & 0.28 & 0.603 & 0.09 & 0.765 \\
\hline D0:UBD-CA & 1.61 & 0.216 & 0.00004 & 0.995 \\
\hline D4:SH-UBD & 0.01 & 0.945 & 0.15 & 0.702 \\
\hline $\mathrm{D} 4: \mathrm{SH}-\mathrm{CA}$ & 60.16 & $<0.001$ & 37.72 & $<0.001$ \\
\hline D4:UBD-CA & 58.18 & $<0.001$ & 31.47 & $<0.001$ \\
\hline D8:SH-UBD & 1.27 & 0.271 & 0.59 & 0.452 \\
\hline D8:SH-CA & 105.19 & $<0.001$ & 74.55 & $<0.001$ \\
\hline D8:UBD-CA & 123.07 & $<0.001$ & 83.92 & $<0.001$ \\
\hline SH:D0-D4 & 0.16 & 0.696 & 0.01 & 0.929 \\
\hline SH:D0-D8 & 2.15 & 0.155 & 0.03 & 0.856 \\
\hline UBD:D0-D4 & 2.15 & 0.155 & 0.97 & 0.334 \\
\hline UBD:D0-D8 & 0.03 & 0.874 & 0.24 & 0.632 \\
\hline CA:D0-D4 & 91.90 & $<0.001$ & 69.67 & $<0.001$ \\
\hline CA:D0-D8 & 87.76 & $<0.001$ & 75.43 & $<0.001$ \\
\hline
\end{tabular}

Abbreviations: period, period of observation; SH, sham males; UBD, urinary bladder damaged males; CA, castrated males; D0, day 0; D4, day 4; D8, day 8.

(without tissue removal) were done $3 \mathrm{~mm}$ from the basis of the insertion of gonads, without affecting gonadal functioning. In the sham operated treatment, the ventral incision was made and sutured without either castration or urinary bladder damage. In all treatments the ventral incision was sutured using sterile synthetic absorbable braided polyglycolic acid suture (USP 5/0, 3/8 circle DS $19 \mathrm{~mm}$, Surgicril ${ }^{\circledR}$, Ref. 11101519). After recovery from anaesthesia operated fish were returned to their experimental aquarium. No mortalities or secondary infections were recorded.

\subsection{Behavioral observations}

Daily behavioral observations ( $10 \mathrm{~min} /$ day) were performed from day 0 (before surgery) to day 8 using instantaneous sampling (sensu Martin and Bateson, 2007) with a 10 s sample interval between scans, in which courtship and aggressive behavior (bite) directed towards females were recorded. Nest presence and body coloration were also recorded daily by direct visual inspection during the observation period. At day 9 the behavior directed by the focal male towards the stimulus male was recorded using continuous recording (sensu Martin and Bateson, 2007), in which the following behavioral patterns were noted: bite, displays, and courtship (for an ethogram of this species see Baerends and Baerends-van Roon (1950)).

\subsection{Quantification of androgens}

Plasma samples $(100 \mu \mathrm{l})$ were extracted with diethyl-ether $(2 \times 4 \mathrm{ml})$ by freezing the aqueous phase in liquid nitrogen $\left(10 \mathrm{~min},-80^{\circ} \mathrm{C}\right)$ after mixing and centrifugation $(1000 \mathrm{~g}, 10 \mathrm{~min}$, $4{ }^{\circ} \mathrm{C}$ ), followed by decanting the supernatant and evaporating it under a stream of nitrogen gas.

Androgen concentrations were measured by radioimmunoassay. The testosterone and 11-ketotestosterone antisera were kindly donated, respectively by Drs. A.P. Scott and David Kime. The testosterone antiserum cross-reactions were $63 \%$ for androstenedione, $35 \%$ for 11 -ketotestosterone, $55 \%$ for $11 \beta$-hydroxytes- 

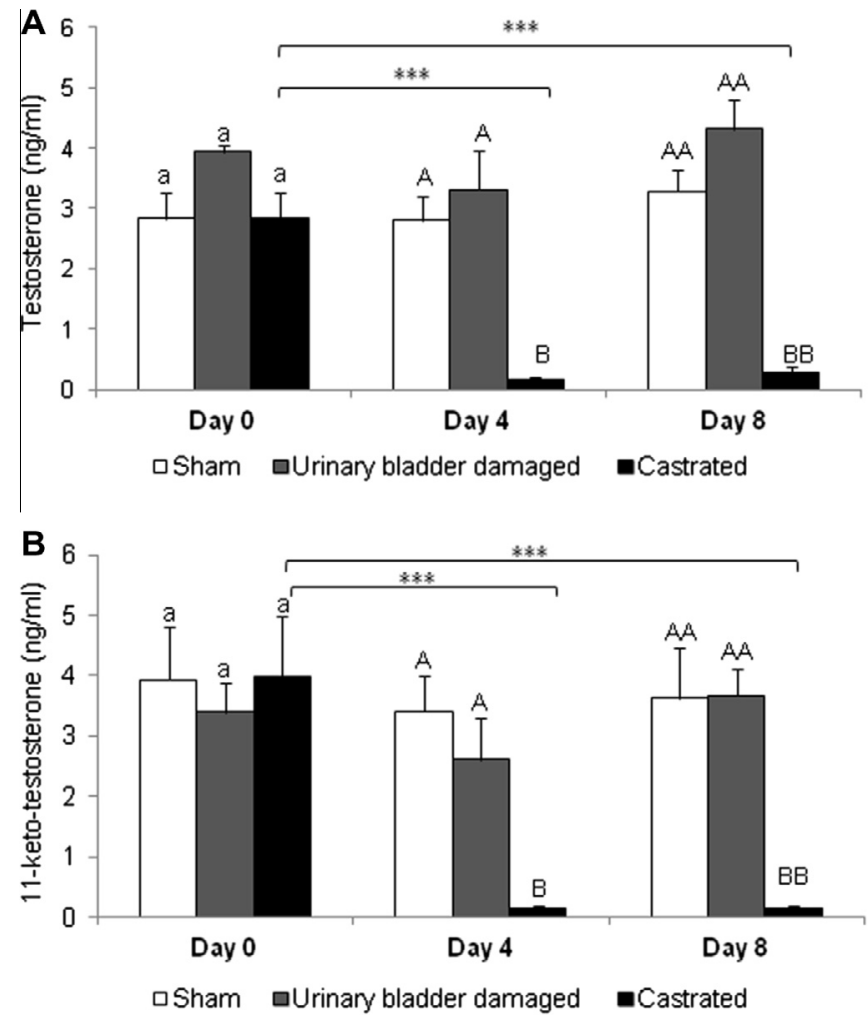

Fig. 3. Blood plasma androgen levels in sham, urinary bladder damaged and castrated males at days 0,4 and 8 (mean \pm standard error): (A) testosterone and (B) 11-ketotestosterone. Significant differences (planned comparisons, $p<0.05$ ) between groups at each sampling point are marked with different letters. Significant differences in androgen levels between pre- and post-surgery sampling points (i.e. day 0 vs. day 4 and day 0 vs. day 8 ) are marked with asterisk.

tosterone, $40 \%$ for $5 \alpha$-androstan- $17 \beta$-ol-3-one, $31 \%$ for $5 \beta$-androstan- $17 \beta$-ol-3-one, $12 \%$ for $5 \beta$-androstane- $3 \alpha, 17 \beta$-diol, $25 \%$ for $5 \alpha$-androstane- $3 \alpha, 17 \beta$-diol. The 11-ketotestosterone antiserum cross-reactions are given elsewhere (Kime and Manning, 1982) and were $20.1 \%$ for $11 \beta$-hydroxytestosterone, $20.6 \%$ for testosterone, $76.9 \%$ for androstenetrione, $30.1 \%$ for $11 \beta$-hydroxyandrostenedione, $52 \%$ for dihydrotestosterone, $3.3 \%$ for cortisol and $1.3 \%$ for cortisone. Tritiated cortisol and testosterone were purchased from GE Healthcare Europe GmbH (Carnaxide, Portugal). The 11ketotestosterone label was produced from tritiated cortisol (Kime and Manning, 1982). Inter- and intra-assay variability was respectively $15.29 \%$ and $3.47 \%$ for testosterone and $9.55 \%$ and $3.58 \%$ for KT.

\subsection{Data analysis}

Data were assessed for the assumptions of parametric statistics (normality and homogeneity of variances, using Levene's test). When data did not conform to these assumptions it was transformed by using the transformation that best corrected the detected deviations. When no transformations yielded satisfactory results non-parametric statistics were used. Following these procedure:

(1) Data for both androgens ( $\mathrm{T}$ and $\mathrm{KT}$ ) were log transformed and subsequently used in a repeated measures ANOVA to test for the main effect of treatment (categorical variable: castrated vs. sham operated vs. urinary bladder damaged) on hormone levels in the experimental period (repeated factor: day 0 vs. day 4 vs. day 8 ); planned comparisons defined a priori were also used to assess: (A) the temporal effect (day 0 vs. day 4 and day 0 vs. day 8 ) of each treatment (castrated, sham operated, urinary bladder damaged) on hormone levels; and (B) the difference between treatments (castrated vs. sham operated vs. urinary bladder damaged) at each sampling point (day 0 , day 4 and day 8 ).

(2) Behavioral data measured by instantaneous sampling (day 1-day 8) did not conform to the assumptions of parametric statistics and therefore non-parametric tests were used; main effect of treatment on the behavioral variables was tested using Kruskall-Wallis tests, followed by post hoc Mann-Whitney tests to contrast each pair of treatments (i.e. sham vs. castration, sham vs. bladder damage, and castration vs. bladder damage).

(3) Behavioral data measured by continuous recording (day 9) fit parametric assumptions (except for \% of time spent in display which was log transformed) and non-transformed raw data was used; a one-way ANOVA was used to test for the effect of treatment (castrated vs. sham operated vs. urinary bladder damaged) on aggressive behavior towards a putative intruder male.

Outliers (diagnosed with Grubber's Test) were handled in two different ways (Cousineau and Chartier, 2010): (1) they were replaced by the mean of the group in the repeated measures ANOVA to avoid losing a whole case; (2) they were removed from the analysis in the non-parametric analysis and in the one-way ANOVAs.

Initial sample sizes were as follows: sham-operated males $=10$; urinary bladder damaged males $=9$, castrated males $=9$. However, sample size may vary across days because of the removal of outliers. One sample was lost during $\mathrm{T}$ analysis.

The effect of treatment on courtship and male aggression towards females was analyzed by using the mean value of post-

Table 2

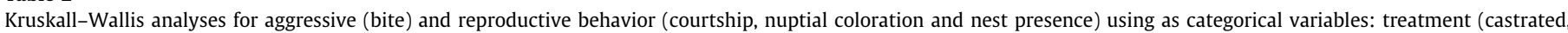
urinary bladder damaged and castrated) measured during day 1-day 8. Planned comparisons using Mann-Whitney $U$ tests are also presented.

\begin{tabular}{|c|c|c|c|c|c|c|c|c|}
\hline & \multicolumn{4}{|c|}{ Aggressive behavior } & \multicolumn{4}{|c|}{ Reproductive behavior } \\
\hline & \multicolumn{2}{|l|}{ Bite } & \multicolumn{2}{|c|}{ Courtship } & \multicolumn{2}{|c|}{ Coloration } & \multicolumn{2}{|c|}{$\underline{\text { Nest presence }}$} \\
\hline & $H$ & $p$-Value & $H$ & $p$-Value & $H$ & $p$-Value & $H$ & $p$-Value \\
\hline \multicolumn{9}{|c|}{ Kruskall-Wallis test } \\
\hline \multirow[t]{2}{*}{ Treatment } & 0.54 & 0.763 & 11.81 & $<0.01$ & 7.54 & $<0.05$ & 15.29 & $<0.001$ \\
\hline & $z$-Value & $p$-Value & $z$-Value & $p$-Value & $z$-Value & $p$-Value & $z$-Value & $p$-Value \\
\hline \multicolumn{9}{|c|}{ Mann-Whitney U test } \\
\hline SH-UBD & 0.22 & 0.825 & 1.20 & 0.230 & 0.52 & 0.60 & 0.000 & 1.00 \\
\hline $\mathrm{SH}-\mathrm{CA}$ & 0.18 & 0.86 & 2.90 & $<0.01$ & 2.80 & $<0.01$ & 3.63 & $<0.001$ \\
\hline UBD-CA & 0.75 & 0.45 & 2.12 & $<0.05$ & 2.01 & $<0.05$ & -2.74 & $<0.01$ \\
\hline
\end{tabular}

Abbreviations: SH, sham males; UBD, urinary bladder damaged males; CA, castrated males. 
surgery behavior (days 1-8). Presence of nest and nuptial coloration were analyzed as \% number over the period of days (for a total of 8 days) after surgery.

Two-tailed tests with a significance of $p<0.05$ were used in all tests. All statistical analyses were performed using the software package Statistica v.7 (StatSoft Inc., Tulsa, Oklahoma, USA).

\section{Results}

\subsection{Effects of castration on androgen levels}

There were significant main effects of both treatment (castrated vs. sham operated vs. urinary bladder damaged) and sampling period (day 0 vs. day 4 vs. day 8 ) on androgen levels (Table 1, Fig. 3A and B). There was also an interaction between treatment and sampling period (Table 1). There were no differences in androgens levels (both $\mathrm{T}$ and KT) among the treatments before surgery (day 0 ). However, both at day 4 and at day 8 after surgery androgen levels were significantly lower in the castrated treatment than either in the sham operated or urinary bladder damaged treatments (Table 1, Fig. 3A and B). Similarly, androgen levels decreased with surgery in the castrated group, but not either in the sham operated or urinary bladder damaged groups (Table 1, Fig. 3A and B).

\subsection{Effects of castration on reproductive and aggressive behavior}

A main effect of treatment was found on all reproductive behaviors: courtship, presence of nests, and nuptial coloration (Table 2, Fig. 4). In contrast to sham or urinary bladder damaged males, courtship was never observed in castrated males. The frequency of courtship behavior did not differ between sham and urinary bladder damaged males (Table 2, Fig. 4A). After surgery nest presence was observed on $65 \%$ of the days for both sham and urinary bladder damaged males, but no nests were built by castrated males (Table 2, Fig. 4B). Among castrated males nuptial coloration was observed just once and for few seconds in one male in a single day. In contrast, nuptial coloration was often observed in males from the sham and urinary bladder damaged treatments (Table 2 , Fig. 4C).

Male aggression towards females was not significantly different across treatments (Table 2; Fig. 4D). Males of all treatments responded equally aggressively towards the neighboring male during the social challenge test on day 9 (frequency: bite, $F_{2,25}=0.75$, $p=0.481$, display, $F_{2,25}=0.47, p=0.629$; percent of time: display, $F_{2,25}=0.56, p=0.578$ Fig. 5). Three out of 10 sham males and 3 out 9 urine bladder damaged males exhibited courtship towards the neighbor male, whereas castrated males never exhibited this behavior.

\section{Discussion}

Castration in Mozambique tilapia decreased circulating levels of androgens in $90 \%$ for T and almost in $100 \%$ for KT (to below $100 \mathrm{pg} /$ $\mathrm{ml}$ ). Concomitantly, courtship behavior, nest building and nuptial coloration were abolished in castrated males, whereas aggressive behavior was not significantly affected. The data from the urinary bladder males confirms the effect of castration on androgens since males from this treatment were similar to those of the sham operated group.

The reduction of androgen levels in castrated males has been previously demonstrated in many species and confirms role of the testis as the main source of androgens in tilapia. The extinction of the whole set of reproductive behaviors (i.e. nuptial coloration, nest building and courtship) in castrated males that experience a
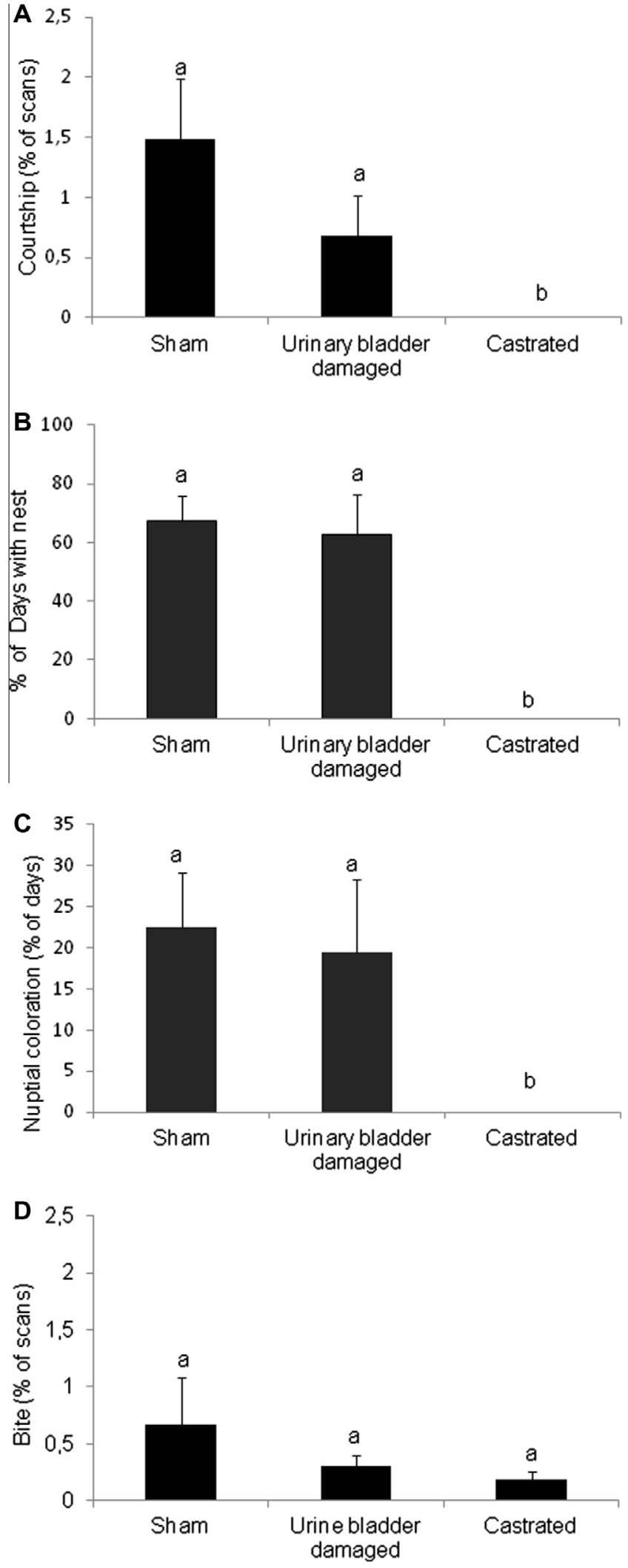

Fig. 4. Percentage of time (mean percentage of days 1-8 \pm SE) that males exhibited reproductive and aggressive behaviors towards females: (A) courtship (sham, $n=10$; urinary bladder damaged, $n=8$; castrated, $n=9$ ); (B) nest presence (sham, $n=10$; urinary bladder damaged, $n=9$; castrated, $n=9$ ); (C) nuptial coloration (sham, $n=10$; urinary bladder damaged, $n=9$; castrated, $n=8$ ) and (D) aggression (sham, $n=9$; urinary bladder damaged, $n=8$; castrated, $n=9$ ). Groups that differed significantly according to Mann-Whitney $U$ tests are marked with different letters. 

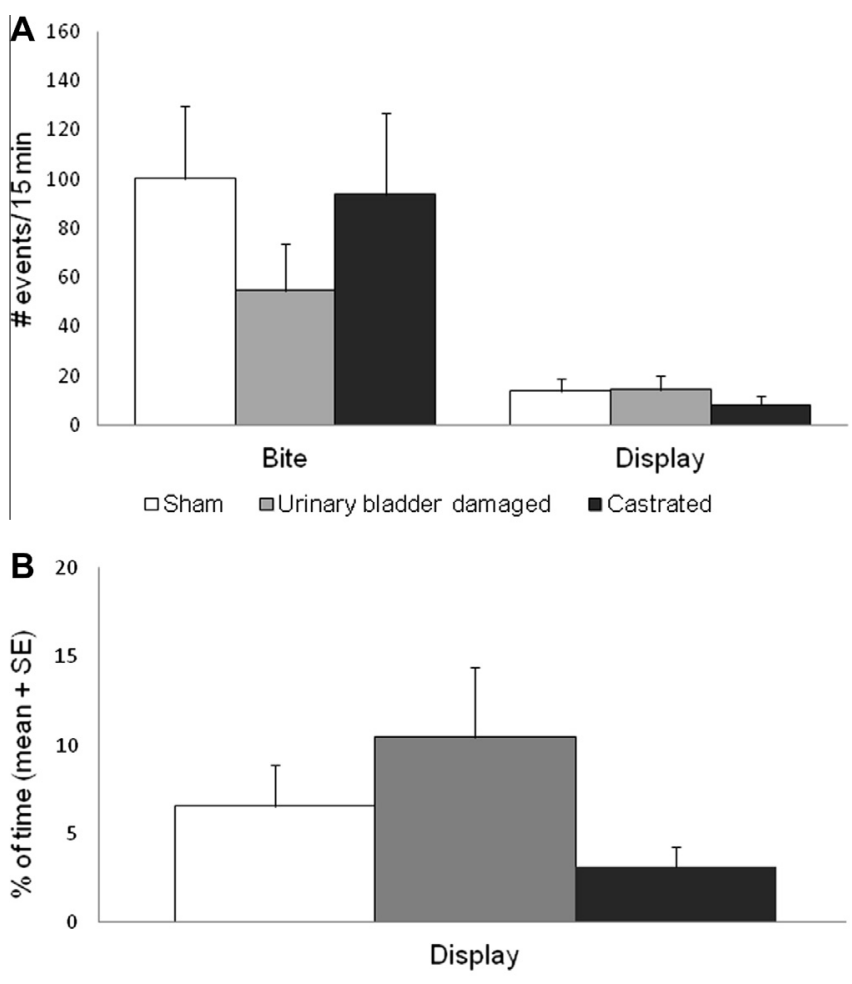

$\square$ Sham 口Urine badder damaged $\quad$ Castrated

Fig. 5. Frequency (A) and duration (B) of aggressive behaviors directed towards the neighboring male during the social challenge at day 9 (mean number of events in $15 \min \pm \mathrm{SE}$ ). Frequency: bite, sham, $n=10$; urinary bladder damaged, $n=9$; castrated, $n=9$; displays, sham $n=10$; urinary bladder damaged, $n=8$; castrated, $n=9$. Percent of time of displays exibhition (sham, $n=10$; urinary bladder damaged, $n=8$; castrated, $n=9$ ).

major reduction in circulating androgen levels further supports a key role for androgens produced in the testis in the linkage between gonadal maturation and the expression of reproductive behavior. The lack of effects of castration on aggressive behaviors apparently contrasts with previous studies in this species where circulating androgens have been shown to be correlated with dominant behavior (Oliveira et al., 1996) and to mediate experiencedependent effects on male competitive behavior (Oliveira et al., 2009). However, these apparently contradictory results can be explained by androgens playing either a mediator or a moderator role in the regulation of aggressive behavior, since only a mediator role would predict androgens to be necessary and sufficient. In other words the data presented here that demonstrates that androgens ( $\mathrm{T}$ and KT, at least) are not necessary for the expression of aggressive behavior do not rule out a moderator role for androgens on the expression of aggressive behaviors. This hypothesis needs further examination with castration followed by androgen replacement therapy. However, the variation in the effects of castration on aggressive behavior within the same species reported in the literature for individuals under different environmental conditions or reproductive state may provide some light. For example, in prenesting three-spined stickleback males, castration and consequently low androgen levels had no effect under short-days and increased aggression under long days (Hoar, 1962) whereas it decreased aggressive behavior in parental males (Wootton, 1970; Baggerman, 1966). Moreover, a meta-analysis on the effects of exogenous administration of androgens on reproductive and aggressive behaviors across vertebrates showed a wide variation in the magnitude of the effect, with larger effects on promiscuous and non-paternal species than on monogamous and paternal (Hirschenhauser and Oliveira, 2006). Altogether, these data support a moderator, rather than a mediator, role of androgens on the expression of aggressive behavior.

The fact that castration did not affect aggressive behavior while abolishing reproductive behaviors also indicates a decoupling of the control mechanisms for the two types of behaviors, with the putative presence of independent reproductive and aggressive neural circuits, and a compartimentalization of the androgen effects on these two modules. This compartimentalization can be achieved for example by the differential expression of androgen receptors between the two modules, and allows for independent responses of the aggressive and the reproductive neural modules to the same circulating levels of androgens. This putative variation in target tissue androgen sensitivity provides a mechanism for independent evolution of reproductive and aggressive behaviors in this species. According to this hypothesis the two modules would escape the pleiotropic-like effects that would be imposed by a similar response to a shared endocrine signal, androgens in this case. This scenario conforms to the "evolutionary potential hypothesis", that postulates that endocrine dependent traits have higher evolvability if selection acts on target tissue sensitivity than on circulating levels of the endocrine signal (Oliveira, 2009).

\section{Conclusion}

In summary, it was demonstrated that androgens are necessary for the expression of reproductive behaviors but not for the expression of aggressive behavior in male tilapia, suggesting a mediator role in the former case and a moderator role in the later. Future studies combining castration with the exogenous administration of different androgens are needed to further explore the moderator role of androgens on aggressive behavior in this species.

\section{Acknowledgments}

The authors thank Elsa Couto for running the radioimmunoassays in the lab. This study was funded by the research Grants PTDC/PSI/71811/2006 and PTDC/MAR/72117/2006 from Science and Technology Foundation, Ministry of Education and Science (FCT, Portugal; Grant holder: R. Oliveira), the European Commission FEDER Program, and the FCT Pluriannual Program (R\&D unit MAR-LVT-Lisboa-331; Grant holder: R. Oliveira). During this project O. Almeida was being supported by a Ph.D. fellowship from FCT (SFRH/BD/37187/2007).

\section{References}

Almeida, O.G., Miranda, A., Frade, P., Hubbard, P.C., Barata, E.N., Canário, A.V.M. 2005. Urine as a social signal in the mozambique tilapia (Oreochromis mossambicus). Chem. Senses 30 (Suppl. 1), i309-i310.

Baerends, G.P., Baerends-van Roon, J.M., 1950. An introduction to the study of the ethology of the cichlid fishes. Behaviour (Suppl.), 1-243.

Baggerman, B., 1966. On the endocrine control of reproductive behaviour in the male three-spined stickleback (Gasterosteus aculeatus L.). In: Symposia of the Society for Experimental Biology, pp. 427-456.

Balthazart, J., Ball, G.F., 1998. New insights into the regulation and function of brain estrogen synthase (aromatase). Trends Neurosci. 21, 243-249.

Barata, E.N., Hubbard, P.C., Almeida, O.G., Miranda, A., Canário, A.V.M., 2007. Male urine signals social rank in the Mozambique tilapia (Oreochromis mossambicus) BMC Biol. 5, 54.

Cousineau, D., Chartier, S., 2010. Outliers detection and treatment: a review. Int. J. Psychol. 3, 58-67.

Desjardins, J.K., Stiver, K.A., Fitzpatrick, J.L., Milligan, N., Kraak, G.J., Balshine, S., 2007. Sex and status in a cooperative breeding fish: behavior and androgens. Behav. Ecol. Sociobiol. 62, 785-794.

Fitzpatrick, J.L., 2005. Male reproductive suppression in the cooperatively breeding fish Neolamprologus pulcher. Behav. Ecol. 17, 25-33.

Foo, J.T.W., Lam, T.J., 1993. Serum cortisol response to handling stress and the effect of cortisol implantation on testosterone level in the tilapia, Oreochromis mossambicus. Aquaculture 115, 145-158.

Gonçalves, D., Oliveira, R., 2010. Hormones and sexual behavior of teleost fishes. In: Norris, D., Lopez, K. (Eds.), Hormones and Reproduction of Vertebrates. Academic Press, pp. 119-147. 
Hirschenhauser, K., Oliveira, R.F., 2006. Social modulation of androgens in male vertebrates: meta-analyses of the challenge hypothesis. Anim. Behav. 71, 265277.

Hirschenhauser, K., Taborsky, M., Oliveira, T., Canàrio, A.V.M., Oliveira, R.F., 2004. A test of the "challenge hypothesis" in cichlid fish: simulated partner and territory intruder experiments. Anim. Behav. 68, 741-750.

Hoar, W.S., 1962. Hormones and the reproductive behaviour of the male threespined stickleback (Gasterosteus aculeatus). Anim. Behav. 10, 247-266.

Kime, D.E., Manning, N.J., 1982. Seasonal patterns of free and conjugated androgens in the brown trout Salmo trutta. Gen. Comp. Endocrinol. 48, 222-231.

Martin, P., Bateson, P., 2007. Measuring Behaviour: An Introductory Guide Cambridge University Press, Cambridge.

Nelson, R., 2005. An Introduction to Behavioural Endocrinology, third ed. Sinauer, Sunderland, MA.

Oliveira, R.F., 2009. Social behavior in context: hormonal modulation of behavioral plasticity and social competence. Integr. Comp. Biol. 49, 423-440.

Oliveira, R., Almada, V.C., 1998. Androgenization of dominant males in a cichlid fish androgens mediate the social modulation of sexually dimorphic traits. Ethology $104,841-858$.
Oliveira, R.F., Gonçalves, D.M., 2008. Hormones and social behaviour of teleost fish. In: Magnhagen, C., Braithwaite, V.A., Forsgren, E., Kapoor, B.G. (Eds.), Fish Behaviour. Science Publishers, Enfield, NH, pp. 61-150.

Oliveira, R.F., Almada, V.C., Canario, A.V.M., 1996. Social modulation of sex steroid concentrations in the urine of male cichlid fish Oreochromis mossambicus. Horm. Behav. 30, 2-12.

Oliveira, R.F., Silva, A., Canário, A.V.M., 2009. Why do winners keep winning? Androgen mediation of winner but not loser effects in cichlid fish. Proc. Biol. Sci. 276, 2249-2256.

Pfaff, D.W., Kordon, C., Chanson, P., Christen, Y. (Eds.), 2008. Hormones and Social Behaviour. Springer Berlin Heidelberg, Berlin, Heidelberg.

Taves, M.D., Desjardins, J.K., Mishra, S., Balshine, S., 2009. Androgens and dominance: sex-specific patterns in a highly social fish (Neolamprologus pulcher). Gen. Comp. Endocrinol. 161, 202-207.

Wong, M., Balshine, S., 2011. The evolution of cooperative breeding in the African cichlid fish, Neolamprologus pulcher. Biol. Rev. Camb. Philos. Soc. 86, 511-530.

Wootton, R.J., 1970. Aggression in the early phases of the reproductive cycle of the male three-spined stikleback (Gasterosteus aculeatus). Anim. Behav. 18, 740746. 